\title{
GREENHOUSE GAS EMISSIONS FROM GLOBAL CITIES
}

Christopher Kennedy ${ }^{1} *$, Julia Steinberger ${ }^{2}$, Barrie Gasson ${ }^{3}$, Yvonne Hansen ${ }^{4}$, Timothy Hillman $^{5}$, Miroslav Havránek ${ }^{6}$, Diane Pataki ${ }^{7}$, Aumnad Phdungsilp ${ }^{8}$, Anu Ramaswami ${ }^{9}$, Gara Villalba Mendez ${ }^{10}$.

1. Dept. of Civil Engineering, University of Toronto, Canada (35 St. George Street, Toronto, M5S 1A4; christopher.kennedy@utoronto.ca; +1 416978 5978).

2. Vienna Institute of Social Ecology, IFF, University of Klagenfurt, Austria (Schottenfeldgasse 29, 1070 Vienna; julias@alum.mit.edu; ++43-1-5224000-411).

3. School of Architecture and Planning, University of Cape Town, South Africa (7701 Rondebosch, Cape Town; Barrie.Gasson@uct.ac.za; +27 21 650-2366).

4. African Centre for Cities, University of Cape Town, South Africa (7701 Rondebosch, Cape Town; Yvonne.Hansen@uct.ac.za; +27 21650 5903)

5. Dept. of Civil Engineering, University of Colorado Denver, USA (Campus Box 113, P.O. Box 173364, Denver, CO 80217-3364; hillman@ colorado.edu; 303 556-4734 ).

6. Charles University Environment Center, Czech Republic (U Krize8, 15000 Prague; Miroslav.Havranek@czp.cuni.cz; +420251080351)

7. Dept. of Earth System Science and Dept. of Ecology \& Evol. Biology, University of California, Irvine, USA (Irvine, CA 92697-3100; dpataki@uci.edu; +1 949-824-9411).

8. Dept. of Energy Management, Dhurakij Pundit University, Thailand (110/1-4

Prachachuen Rd., Laksi, Bangkok 10210, aumnad@dpu.ac.th; +662 9547300 Ext. 587). 9. Dept. of Civil Engineering, University of Colorado Denver, USA (Campus Box 113, P.O. Box 173364, Denver, CO 80217-3364; Anu.Ramaswami@cudenver.edu; 303 5564734 ).

10. Institut de Ciència i Tecnologia Ambientals, Autonomous University of Barcelona (Edifici Cn 08193 Bellaterra; gara.villalba@uab.es; +93.581.24.88).

\section{Supporting Information}

This supporting information provides detailed GHG emissions by sub-sectors: S1 electricity; S2 heating and industrial fuels; S3 road transportation; S4 waste; S5 aviation, marine and industrial processes. 


\begin{tabular}{lcccc}
\hline & $\begin{array}{c}\text { Electricity } \\
\text { consumption } \\
(\mathrm{GWh})\end{array}$ & $\begin{array}{c}\text { Per capita } \\
\text { electricity } \\
(\mathrm{MWh} / \text { cap. })\end{array}$ & $\begin{array}{c}\text { GHG intensity } \\
(\mathrm{t} \mathrm{eCO} / \mathrm{GWh}) \\
\text { incl. line loss }\end{array}$ & $\begin{array}{c}\text { GHG emissions } \\
(\mathrm{t} \mathrm{eCO} / \text { cap. })\end{array}$ \\
\hline Bangkok & 28,500 & 5.04 & 550 & 2.77 \\
Barcelona & 7,479 & 4.66 & 143 & 0.67 \\
Cape Town & 12,209 & 3.49 & 969 & 3.38 \\
Denver & 6,659 & 11.49 & 792 & 9.10 \\
Geneva & 2,793 & 6.46 & 54 & 0.35 \\
London & 39,237 & 5.33 & 469 & 2.50 \\
Los Angeles & 63,919 & 6.71 & 368 & 2.46 \\
New York City & 49,567 & 6.07 & 497 & 3.01 \\
Prague & 5,506 & 4.66 & 710 & 3.31 \\
Toronto & 55,778 & 10.04 & 246 & 2.47 \\
\hline
\end{tabular}

Table S1. Electricity use, GHG intensity and emissions 


\begin{tabular}{|c|c|c|c|c|}
\hline & $\begin{array}{c}\text { Energy } \\
\text { consumption } \\
\text { TJ }\end{array}$ & Fuel types & $\begin{array}{l}\text { Per capita } \\
\text { consumption } \\
\text { GJ/cap. }\end{array}$ & $\begin{array}{c}\text { GHG emissions } \\
\text { t eCO } \mathrm{eC}_{2} / \text { cap. }\end{array}$ \\
\hline Bangkok & 160,530 & $\begin{array}{l}\text { Bagasse (21\%); Fuel Oil (20\%); Lignite (14\%); Coal } \\
\text { /coke (13\%); Natural Gas (10\%); LPG ( } 8 \%) \text {; Rice Husk } \\
(5 \%) \text {; Wood (5\%); Diesel }(4 \%)\end{array}$ & 28.4 & 2.49 \\
\hline Barcelona & 24,147 & Natural Gas (96\%); LPG (4\%) & 15.0 & 0.85 \\
\hline Cape Town & 54,959 & $\begin{array}{l}\text { Gasoil (37\%); Heavy Furnace Oil (22\%); LPG (18\%); } \\
\text { Paraffin (15\%); Coal (7\%); Other }(1 \%)\end{array}$ & 15.7 & 1.15 \\
\hline Denver & 42,622 & Natural Gas $(\sim 100 \%)$ & 73.5 & 4.12 \\
\hline Geneva & 22,163 & Fuel Oil (58\%); Natural Gas (40\%); Other (2\%) & 51.3 & 3.45 \\
\hline London & 330,648 & Natural Gas (91\%); CHP (6\%); Oil (4\%) & 44.9 & 2.58 \\
\hline Los Angeles & 229,985 & Natural Gas (94\%); Petroleum (6\%) & 24.2 & 1.37 \\
\hline New York City & 414,858 & $\begin{array}{l}\text { Natural Gas (61\%); Distillate Fuel Oil (19\%); CHP } \\
(11 \%) ; \text { Residual Fuel Oil (8\%); Kerosene }(1 \%)\end{array}$ & 50.8 & 3.13 \\
\hline Prague & 55,264 & $\begin{array}{l}\text { Natural Gas }(68 \%) \text {; Heat Pipeline (17\%); Coal (15\%); } \\
\text { Fuel Oil }(0.4 \%)\end{array}$ & 46.8 & 3.20 \\
\hline Toronto & 327,000 & Natural Gas $(\sim 100 \%)$ & 58.9 & 3.30 \\
\hline
\end{tabular}

Table S2. GHG emissions from heating and industrial fuel use 


\begin{tabular}{lccc}
\hline & $\begin{array}{c}\text { Gasoline } \\
\text { consumption } \\
\text { ML }\end{array}$ & $\begin{array}{c}\text { Diesel } \\
\text { consumption } \\
\text { ML }\end{array}$ & $\begin{array}{c}\text { GHG emissions } \\
\text { t eCO } / \text { cap. }\end{array}$ \\
\hline Bangkok & 2,741 & 2,094 & 2.27 \\
Barcelona & 209 & 266 & 0.77 \\
Cape Town & 1,249 & 724 & 1.44 \\
Denver & 1,234 & 197 & 6.31 \\
Geneva & 260 & 51 & 1.85 \\
London & 1,797 & 1,238 & 1.22 \\
Los Angeles & 14,751 & 3,212 & 4.92 \\
New York City & 4,179 & 657 & 1.53 \\
Prague & 357 & 281 & 1.44 \\
Toronto & 6,691 & 2,011 & 4.05 \\
\hline
\end{tabular}

Table S3. Ground Transportation Fuels, consumption and GHG emissions 


\begin{tabular}{lcccc}
\hline & $\begin{array}{c}\text { Incinerated } \\
\text { Waste } \\
\text { (t/cap. })\end{array}$ & $\begin{array}{c}\text { Landfill } \\
\text { Waste } \\
\text { (t/cap. })\end{array}$ & $\begin{array}{c}\text { DOC of landfill } \\
\text { waste } \\
\text { (t C / t waste) }\end{array}$ & $\begin{array}{c}\text { GHG emissions } \\
\text { (t eCO } / \text { cap. })\end{array}$ \\
\hline Bangkok & 0 & 0.806 & 0.18 & 1.23 \\
Barcelona & 0.19 & 0.17 & 0.17 & 0.24 \\
Cape Town & 0 & 0.75 & 0.28 & 1.78 \\
Denver & 0 & 1.25 & 0.22 & 0.59 \\
Geneva & 0.74 & 0.35 & 0.01 & 0.38 \\
London & 0 & 1.40 & 0.07 & 0.21 \\
Los Angeles & 0 & 1.20 & 0.19 & 0.49 \\
New York City & 0 & 0.971 & 0.17 & 0.35 \\
Prague & 0.17 & 0.046 & 0.19 & 0.11 \\
Toronto & 0.012 & 0.736 & 0.21 & 0.33 \\
\hline
\end{tabular}

Table S4. GHG emissions from Waste. Methane emissions from landfill waste are long-term releases over several years. $\mathrm{DOC}=$ degradable organic content 


\begin{tabular}{lccc}
\hline & Air & Marine & $\begin{array}{c}\text { Direct } \\
\text { industrial }\end{array}$ \\
& $\mathrm{t} \mathrm{eCO} / \mathrm{cap}$. & $\mathrm{t} \mathrm{eCO}_{2} / \mathrm{cap}$. & $\mathrm{t} \mathrm{eO}_{2} / \mathrm{cap}$. \\
\hline Bangkok & 1.92 & unknown & unknown \\
Barcelona & 1.66 & unknown & unknown \\
Cape Town & 0.89 & 2.92 & unknown \\
Denver & 1.42 & 0 & unknown \\
Geneva & 1.72 & 0 & unknown \\
London & 3.12 & negligible & unknown \\
Los Angeles & 1.86 & 1.69 & 0.22 \\
New York City & 1.74 & 0.76 & unknown \\
Prague & 0.90 & 0 & 0.43 \\
Toronto & 0.83 & negligible & 0.57 \\
\hline
\end{tabular}

Table S5. GHG emissions from other sources 